\title{
FACTORES CLAVE EN EL ÉXITO DE LAS EMPRESAS AGRARIAS: EL CASO DE LAS COOPERATIVAS HORTOFRUTÍCOLAS EN ESPAÑA
}

\author{
Vanessa Campos-Climent ${ }^{1}$, Joan Ramon Sanchis-Palacio ${ }^{2}$
}

\section{RESUMEN}

El tamaño es una variable contingente que puede influir en los resultados de las empresas. En este trabajo se pretende contrastar la existencia o no de una relación significativa entre tamaño y resultados empresariales de las empresas agrarias, en particular de las cooperativas hortofrutícolas españolas. Para ello, se realiza un estudio empírico en base a la información económica y financiera obtenida de los registros oficiales de las cooperativas agrarias dedicadas a la comercialización de frutas y hortalizas sobre el cual se ha aplicado un análisis de regresión. El estudio demuestra que no existe una relación significativa positiva entre los resultados empresariales y el tamaño de este tipo de entidades, por lo que se puede afirmar que la capacidad competitiva de una empresa agraria depende de su propia capacidad de gestión y no de un factor de contingencia como el tamaño.

Palabras clave: Tamaño, Resultados empresariales, Sector agrario, Cooperativas hortofrutícolas.

JEL: M10, Q13

1. Doctora en Economía Social, Universitat de Valencia, Docente Departamento de Dirección de Empresas, Universitat de Valencia. Email: vanessa.campos@uv.es, España.

2. Doctor en Economía de la empresa, Universitat de Valencia, Catedrático Departamento de Dirección de Empresas, Universitat de Valencia. Email: joan.r.sanchis@uv.es, España. 


\title{
KEY FACTORS FOR SUCCESS IN AGRIFOOD FIRMS. THE CASE OF SPANISH FRUIT \& VEGETABLES COOPERATIVES
}

\author{
By: Vanessa Campos-Climent, Joan Ramon Sanchis-Palacio
}

\begin{abstract}
Size is a contingency variable that can influence firms' performance. This study aims to contrast whether exists or not a significant relationship between organizational size and performance in agrifood firms, particularly in the case of Fruit and Vegetables Cooperatives in Spain. Therefore, an applied empirical study using regression analysis is carried out from economic and financial information of such companies obtained from official registers. The study shows that there is not a significant positive relationship between performance and size for such type of firms.
\end{abstract}

Key words: Size, Performance, Agrifood firms, Fruit and Vegetable (F\&V) Cooperatives.

JEL: M10, Q13. 


\title{
OS FATORES-CHAVE NO SUCESSO DA AGRICULTURA EMPRESAS: O CASO DA COOPERATIVA HORTICULTURAL EM ESPANHA
}

\author{
Por: Vanessa Campos-Climent, Joan Ramon Sanchis-Palacio
}

O tamanho da cota é uma variável que pode influenciar os resultados das empresas. Este trabalho tem como objetivo contrastar a existência de uma relação significativa entre tamanho e desempenho dos negócios das empresas agrícolas, em especial as cooperativas hortícolas espanhóis. Para fazer isso, um estudo empírico é realizado com base em informações obtidas dos registros oficiais das cooperativas agrícolas que se dedicam à comercialização de frutas e legumes de informação económica e financeira sobre a qual foi aplicada uma análise de regressão. $O$ estudo mostra que há uma relação positiva e significativa entre o desempenho da empresa e do tamanho de tais instituições, para que possamos dizer que a competitividade de uma empresa agrícola depende da sua própria capacidade de gestão e não um fator contingência como tamanho.

Palavras-chave: tamanho, Os resultados do negócio, Setor agrícola, Cooperativas hortícolas.

JEL: M10, Q13. 


\section{INTRODUCCIÓN}

Las cooperativas agrarias tienen sus antecedentes en el asociacionismo agrario $^{3}$, que ha tenido como objetivo conseguir las mejores condiciones, en precios y rentabilidad, para los agricultores. Así, las cooperativas agrarias son reconocidas como la base para la estructuración del sistema agroalimentario y un instrumento necesario para la cohesión del mundo rural (Cervera y Bohórquez, 2000: 35; Bahamonde, 2000: 43; Ruiz, Hernández y García, 2006: 72).

Es la primera fuerza asociativa organizada empresarialmente de pequeños y medianos agricultores a nivel mundial y según datos de la Confederación General del Cooperativismo Agrario en la Unión Europea COGECA, en Europa representan el $60 \%$ de la recolección, comercialización y transformación en origen de la producción agraria. Su peso es del 1,2\% del PIB y del 5,4\% del empleo en la UE-25 e incluye a más de 32.000 empresas cooperativas, que integran once millones de explotaciones con una cifra de facturación de más de 250.000 millones de euros (COGECA, 2010: 41-47). Las cooperativas agrarias en España representan una de las primeras entidades de la Economía Social (Barea y Monzón, 2002: 125-137; Monzón, 2009: 180-191) y según Cooperativas Agro-alimentarias ${ }^{4}$ son un total de 3.989 entidades, con una cifra de facturación de casi 19.000 millones de euros, 94.156 personas empleadas y 1.160 .337 socios.

En la actualidad, las cooperativas agrarias representan una opción válida ante la situación de crisis económica a la que se enfrenta la agricultura mediterránea y realizan una función relevante dentro del entorno rural en el que se desenvuelven (Juliá y Marí, 2002: 28; Ruiz, Hernández y Garcia, 2006: 74). El desarrollo del cooperativismo hortofrutícola ha sido importante históricamente. Por una parte, la agrupación en cooperativas de comercialización de productos agrarios contrarresta, en parte, el fuerte poder de negociación que tienen las empresas de distribución comercial dentro de mercados oligopsónicos donde los clientes imponen los precios. Por otra parte, la creación de cooperativas de explotación comunitaria de la tierra permite abaratar en parte los elevados costes de los factores productivos, en especial, los correspondientes a la mano de obra (Fernández-Zamudio et al., 2006: 201; Valentinov, 2007: 59). Sin embargo, la existencia de unos precios de los productos hortofrutícolas bajos y de unos costes productivos fijos altos, unido a la situación de crisis económica y financiera iniciada en el verano del 2007, afectan negativamente a la situación económica y financiera del sector agrario en general (Bahamonde, 2000: 47; Fernández Zamundio et al., 2006: 208) y de las cooperativas hortofrutícolas en particular (Domingo Sanz, 2001: 177; Galdeano, 2006: 199; Montegut, 2006: 101; Montegut y Cristobal, 2010: 41; Giagnocavo et al., 2012: 89).

El cooperativismo agrario, con el paso del tiempo, ha conseguido un reconocimiento, no solo en España, sino también a nivel internacional (Rojas, 2007: 63). Pero como señalan Meliá y Juliá (2008: 58), con los nuevos parámetros en los que

3. Los primeros antecedentes del asociacionismo agrario en España lo representan los sindicatos agrarios (Marí y Juliá, 2001: 61-63).

4. Esta entidad representa a la mayoría de las cooperativas agrarias de España y anteriormente se denominaba Confederación de Cooperativas Agrarias de España CCAE. 
se basa la economía mundial, ¿̇tienen las cooperativas posibilidad de continuar desarrollando su actividad?; y si es así, ¿cuál es el papel al que están llamadas a llevar a cabo en este nuevo escenario?. Su desarrollo está condicionado, según Montegut y Cristóbal (2010: 45), por la intensidad de la concentración de la oferta. Las exigencias de las empresas de distribución, de los consumidores y de las Administraciones Públicas respecto a la trazabilidad, seguridad alimenticia y respecto al medio ambiente, han comportado una reorganización en profundidad de los sistemas agroalimentarios (Filippi y Triboulet, 2006: 27).

Por todo lo apuntado, se hace necesario un estudio sobre la viabilidad económica y financiera de las cooperativas hortofrutícolas con el objetivo de analizar cuál es su situación económica y financiera actual y sus perspectivas de futuro (Soboh et al., 2012: 148). Desde este planteamiento, resulta de interés analizar si determinados parámetros estructurales de la organización como su tamaño, son claves en la mejora de la eficacia y la eficiencia de estas entidades. El estudio se enmarca, por tanto, dentro del enfoque contingente de las organizaciones (Mintzberg, 1979: 230), aspecto que ha sido analizado por una gran cantidad de investigadores en Dirección de Empresas (Claver et al., 2011: 9).

La aportación de este trabajo, además de ofrecer otra evidencia empírica más al respecto, reside en aplicar el enfoque contingente al caso de las cooperativas hortofrutícolas, siendo en este sentido el primer trabajo realizado en España sobre este tipo de organizaciones. El presente trabajo tiene como objetivo demostrar si determinados parámetros estructurales o factores de contingencia como el tamaño de la organización, influyen positivamente en la mejora de los resultados de estas entidades. La metodología consistirá en la realización de un estudio empírico, consistente en la aplicación de un análisis de regresión entre la variable independiente (parámetros de estructura) y la variable dependiente (resultados empresariales) a partir de la información económica y financiera obtenida de los registros oficiales de Cooperativas. El trabajo se ha estructurado en cinco apartados, incluido este primero de introducción. En el segundo apartado se define el marco teórico del trabajo y se caracterizan las cooperativas hortofrutícolas objeto de estudio. En el tercer apartado se describe el diseño del estudio empírico realizado, incluyendo su metodología, objetivos e hipótesis a contrastar. En el cuarto apartado se analizan los resultados obtenidos del estudio empírico realizado y, por último, en el quinto apartado se incluyen las conclusiones obtenidas.

\section{TAMAÑO Y RESULTADOS EMPRESARIALES. SU REPERCUSIÓN EN LAS COOPERATIVAS HORTOFRUTÍCOLAS}

El éxito de una estrategia depende, en gran medida, de los factores de contingencia que condicionan el funcionamiento de una organización, es decir, de variables estructurales como el tamaño, el entorno o la tecnología. Esto es lo que se conoce como el fit externo o ajuste estrategia-estructura (Miles y Snow, 1978). Según estos pensadores económicos, cada estrategia requiere diferentes formas de organizar la tecnología, diferentes estructuras y procesos y diferentes políticas de recursos humanos. 
La mayoría de los modelos desde los cuales se analiza el ajuste estrategia-estructura tienen su origen en la teoría y el diseño de las organizaciones (Galbraith, 2002) y es mediante el enfoque contingente como estos modelos llevan a cabo el análisis de la influencia de los diferentes factores de contingencia (entorno, tecnología, tamaño) sobre la estructura organizativa de la empresa (Mintzberg, 1979). De esta manera, se consigue el ajuste o fit interno a través de su hipótesis de configuración. Sin embargo, el ajuste interno no explica por sí solo la eficiencia o el resultado de la empresa, si no que éste tiene que complementarse con el ajuste o fit externo, que es el que hace referencia a la relación entre dichos factores de contingencia y la estrategia de la empresa (Miles y Snow, 1994). Cuando se combinan ambos, el ajuste es completo, convirtiéndose en un proceso mediante el cual los factores de contingencia condicionan la estrategia seleccionada y ésta última a su vez condiciona la estructura (Nadler y Tushman, 1997).

Entre los diferentes factores de contingencia, sobre los que se ha generado mayor literatura son el entorno, el tamaño y la tecnología. La mayor parte de los estudios se han centrado en el análisis de la evidencia empírica sobre la relación entre estos factores y la rentabilidad empresarial. Uno de dichos factores es el tamaño de la organización. Sin embargo, el cuerpo teórico sobre la relación entre tamaño y estructura organizativa es realmente escaso, pues la mayor parte de los estudios publicados al respecto se han centrado en contrastaciones empíricas. En cambio, existen una gran cantidad de trabajos que analizan la relación entre el tamaño y la estrategia empresarial, en especial, la estrategia de crecimiento ${ }^{5}$. En este sentido, existen tanto trabajos que demuestran la existencia de algún tipo de relación positiva entre ambos conceptos (Prais, 1976) como otros que evidencian una relación negativa (Kumar, 1985).

La mayor parte de los trabajos sobre la influencia del tamaño en el ajuste estrategia-estructura, han intentado evidenciar algún tipo de relación entre éste y la eficacia y los resultados empresariales (Miller, 1992). El tamaño empresarial puede condicionar el comportamiento económico-financiero de una organización, lo que afectará a sus rendimientos (Jacquemin y Ghellink, 1980). No obstante, no se ha podido demostrar con rotundidad que el aumento de tamaño implique una mejora en los resultados de la empresa.

Sin embargo, tradicionalmente se ha considerado que cuanto más grande es una empresa, mejores resultados obtiene, sustentado en el hecho que las empresas de gran tamaño tienen mayor acceso a recursos técnicos y de mercado, por lo que es mas fácil que consigan economías de escala, poseen un mayor poder de negociación frente a sus clientes, proveedores y entidades bancarias y tienen mayor facilidad para acceder a los mercados internacionales (Demsetz, 1973) ${ }^{\mathbf{6}}$.

5. La Ley del Efecto Proporcional de Gibrat (1931) describe el crecimiento como el resultado de diversos factores que actúan de forma multiplicativa sobre el tamaño inicial de la empresa (González Pérez y Correa, 1998).

6. Este aspecto requeriría de un análisis más exhaustivo sobre el tema de las economías de escala y el tamaño de las empresas. Sin embargo, las limitaciones del presente trabajo impiden abordar el tema en mayor profundidad. Se puede consultar a Vidal (1999), Sabaté (2002) y Adenaeuer y Heckelei (2011), entre otros. 
Actualmente, existe una gran preocupación por el estudio de la rentabilidad y la eficacia económica de las empresas agrarias (Adenaeuer y Heckelei, 2011), dado que estas se encuentran en una situación de crisis estructural que puede suponer su desaparición a medio plazo (Giagnocavo et al., 2012). La gran mayoría de trabajos empíricos realizados en el sector agrario se han centrado en un estudio descriptivo de ratios para tratar de determinar la situación económica y financiera de estas entidades.

Desde el año 1989 se han publicado en España unos 17 estudios que han aplicado el análisis de ratios al sector agrario (cooperativas agrarias, asociaciones de productores agrarios y empresas agroalimentarias en general), de los cuales 5 corresponden a las cooperativas hortofrutícolas. En Vidal (1999) se analiza la eficiencia económica de las cooperativas de comercialización de la Comunidad Valenciana, Domingo Sanz (2001) estudia la rentabilidad económica de las cooperativas agrarias en Andalucía, Sabaté (2002) analiza la eficiencia económica de las cooperativas de la fruta en Cataluña, Arcas, García Pérez y Guzmán (2006) estudian la eficiencia y el rendimiento económico de las cooperativas agrarias en España y Sarasa, Rivera y Capo (2009) hacen lo propio en las cooperativas agrarias de la Comunidad Valenciana. En todos estos estudios se demuestra que se trata de empresas con unos bajos niveles de rentabilidad y eficiencia, pero ninguno de ellos demuestra que el aumento de tamaño suponga un incremento de la rentabilidad de estas empresas. Sin embargo, existe una literatura teórica abundante que redunda en la necesidad de aumentar el tamaño de este tipo de organizaciones mediante procesos de concentración empresarial, principalmente mediante fusiones, con el fin de reducir sus costes y ser más eficientes (Galdeano, 2006; Meliá y Julia, 2008); no obstante, algunos estudios empíricos sobre fusiones concluyen que no siempre estas operaciones permiten obtener los resultados esperados en cuanto a ingresos, costes y rentabilidad (Meliá, Julia y García, 2007), por lo que se plantean estrategias de crecimiento alternativas como las alianzas (Arcas, 2002).

\section{METODOLOGÍA: MUESTRA, MEDIDAS E HIPÓTESIS}

El estudio empírico ha consistido en relacionar el parámetro de diseño tamaño (variable independiente) con los resultados empresariales (variable dependiente) de las cooperativas hortofrutícolas en España. Partiendo de la población total de estas entidades, se ha seleccionado como muestra para la realización del estudio empírico todas aquellas cooperativas inscritas y calificadas en el Registro Administrativo autonómico correspondiente y que entregan su documentación contable en el Registro de Cooperativas. El motivo de elección de la muestra ha sido la facilidad para acceder a la información económica y financiera de estas entidades, la cual se ha conseguido a través del contacto directo con los Registros de Cooperativas. De esta manera, se ha obtenido información sobre los resultados empresariales de las cooperativas y sobre sus variables de tamaño, al igual que se ha hecho en otros estudios similares (Montegut, 2006) ${ }^{7}$.

7. Las cuentas anuales utilizadas corresponden al formato abreviado de cuentas y actualizados al nuevo Plan General Contable vigente desde el año 2008. 
La muestra seleccionada, según se puede observar en la Tabla 1, supone un porcentaje sobre el total de la población que oscila entre el 33,8\% según el número de socios y el 7,7\% según el número de entidades, lo que supone una muestra estadísticamente representativa. Se han analizado 73 entidades de un total de 945, que poseen 54.200 socios y una cifra de facturación de 537 millones de euros.

Tabla 1. Representatividad de la muestra

\begin{tabular}{|l|c|c|c|}
\hline \multicolumn{1}{|c|}{ Variable } & Población & Muestra & Porcentaje \\
\hline No Entidades & 945 & 73 & 7,72 \\
\hline No Socios & 160.500 & 54.200 & 33,77 \\
\hline Cifra facturación* & 3.700 & 537 & 14,51 \\
\hline
\end{tabular}

* En millones de euros - Fuente: elaboración propia

Según la Tabla 2, las variables objeto de estudio se han agrupado en dos dimensiones globales diferentes que a su vez se componen de una serie de constructos. La dimensión funcionamiento de la organización se ha medido a través del constructo tamaño organizativo, para el cual se ha utilizado una variable multicriterio que combina linealmente elementos económicos, financieros y patrimoniales (Ferruz y González, 1994; González y Correa, 1998). Las variables utilizadas han sido los activos totales, los fondos propios y los ingresos de explotación (González, Hernández y Rodríguez, 2000). No se ha incluido la variable empleados, a pesar de ser una de las más utilizadas en este tipo de estudios, por no disponer de dicha información para todas las empresas. No obstante, la variable multicriterio utilizada es idónea para medir el tamaño de una organización, al considerar diferentes aspectos empresariales en su medición.

Tabla 2. Dimensiones, constructos y variables del estudio

\begin{tabular}{|c|c|c|}
\hline Dimensión & Constructos & Variables \\
\hline $\begin{array}{l}\text { Funcionamiento } \\
\text { de la } \\
\text { Organización }\end{array}$ & Tamaño & $\begin{array}{l}\text { - Activos Totales Medios ATM } \\
\text { - Fondos Propios FP } \\
\text { - Ingresos de explotación IE }\end{array}$ \\
\hline \multirow{3}{*}{$\begin{array}{c}\text { Resultados de la } \\
\text { Organización }\end{array}$} & $\begin{array}{l}\text { Capacidad } \\
\text { de } \\
\text { financiación }\end{array}$ & $\begin{array}{l}\text { - Liquidez } \mathrm{L}=(\text { Activo Corriente-Existencias)/Pasivo } \\
\text { Corriente } \\
\text { - Solvencia S=Activo Total/(PC+PNC) } \\
\text { - Apalancamiento AP=(PC+PNC)/Pasivo Total } \\
\text { - Estructura del Apalancamiento EA=PC/(PC+PNC) }\end{array}$ \\
\hline & $\begin{array}{l}\text { Capacidad } \\
\text { económica }\end{array}$ & $\begin{array}{l}\text { - Margen sobre Ventas MV=BAIT/Ventas } \\
\text { - Rotación de los Activos RA=Ventas/Activo Total } \\
\text { - Eficiencia E=Gastos explotación/BAIT }\end{array}$ \\
\hline & $\begin{array}{l}\text { Capacidad } \\
\text { mixta }\end{array}$ & $\begin{array}{l}\text { - Rentabilidad de los Activos ROA=BAIT/Activo Total } \\
\text { - Rentabilidad de los Recursos Propios ROE=BAT/ } \\
\text { Fondos propios }\end{array}$ \\
\hline
\end{tabular}

PC=Pasivo Corriente; $\mathrm{PNC}=$ Pasivo no Corriente; $\mathrm{BAIT}=$ Beneficio antes de intereses e impuestos; $\mathrm{BAT}=$ Beneficio antes de impuestos.

Fuente: elaboración propia. 
La segunda dimensión (resultados empresariales), inicialmente se ha medido a través de la eficacia económica, que mide el logro de los objetivos económicos y financieros de estas empresas. La eficacia empresarial se ha medido a través de dos variables cuantitativas, como son, la rentabilidad de los activos ROA (beneficio antes de intereses e impuestos por activos totales BAIT/AT) y la rentabilidad de los recursos propios ROE (beneficio antes de impuestos por recursos propios BAT/ $\mathrm{RP}$ ). Entendemos que si este tipo de organizaciones han de ser competitivas en el mercado y esto es realmente lo que se quiere analizar, se han de utilizar variables económicas y financieras para su análisis (Rumelt, 1991). Sin embargo, dado que se ha constatado que en este tipo de organizaciones (cooperativas hortofrutícolas) los valores de la rentabilidad económica están en la mayoría de los casos próximos a cero (porque la cooperativa sacrifica la obtención de beneficio a cambio de ofrecer un servicio de calidad al cliente que es el socio de la cooperativa), se ha considerado oportuno utilizar también otro tipo de medidas de rendimiento como son las ratios financieras para medir la capacidad de financiación de la empresa (liquidez, solvencia, apalancamiento y estructura del apalancamiento) y las ratios económicas para medir la capacidad para generar resultados por parte de la empresa (margen sobre ventas, rotación de los activos y eficiencia).

En ambos casos se ha tomado como medida cuantitativa el valor de la mediana (y no de la media) ${ }^{8}$ correspondiente a los diferentes años de funcionamiento de la empresa dentro del período 2007-2011.

Las hipótesis consideradas en el estudio se derivan del objetivo planteado respecto a si existe o no una relación significativa entre el factor de contingencia tamaño organizativo y los resultados empresariales obtenidos por las cooperativas hortofrutícolas en España. Se considerara la Hipótesis nula Ho o su hipótesis alternativa. Dado que se ha considerado un único factor de contingencia (tamaño), la hipótesis planteada es también una.

Son numerosos los estudios que incluyen el tamaño como variable organizativa y lo relacionan con los resultados organizativos, obteniéndose de ello tanto argumentos a favor de una relación positiva (Youndt et al., 1996) como en contra (Geary, 1999). Existen numerosos trabajos que han demostrado que las variables de tamaño como los activos totales, los fondos propios y los ingresos de explotación no siempre están asociados al éxito empresarial (Giagnocavo et al., 2012: 89). Sin embargo, en general, se considera que las organizaciones de un mayor tamaño utilizan métodos empresariales modernos que propician la obtención de resultados organizativos, dado que la mayor dimensión permite el acceso a recursos y capacidades y a determinadas sinergias y economías de alcance y de escala que se pueden traducir en resultados empresariales positivos (Meliá y Juliá, 2008: 58). En este sentido, se formula la hipótesis:

8. Se ha utilizado esta medida de posición por ser la más representativa de una distribución de frecuencias a escala ordinal, ya que describe la tendencia central y, por tanto, es menos sensible a los valores extremos. 
Hipótesis. El tamaño influye sobre los resultados empresariales de las cooperativas hortofrutícolas, de manera que las empresas de mayor tamaño presentan un mayor éxito organizativo.

No obstante, se ha considerado oportuno desglosar esta hipótesis en una serie de subhipótesis con el fin de comparar la variable tamaño con las diferentes medidas de resultados empresariales utilizadas en nuestro estudio. Así, se han contemplado las siguientes subhipótesis:

Hipótesis 1. El tamaño influye sobre la capacidad de financiación de las cooperativas hortofrutícolas, de manera que las empresas de mayor tamaño presentan una mayor capacidad de financiación.

Hipótesis 2. El tamaño influye sobre la capacidad de generar resultados de las cooperativas hortofrutícolas, de manera que las empresas de mayor tamaño presentan una mayor capacidad de generar resultados.

Hipótesis 3. El tamaño influye sobre la capacidad de influencia de la estructura económica y financiera para generar beneficios de las cooperativas hortofrutícolas, de manera que las empresas de mayor tamaño presentan una mayor capacidad de influencia.

Para la realización del estudio empírico se han utilizado técnicas de análisis multivariante, análisis de regresión y contraste de varianzas. Previamente se ha realizado un análisis de componentes principales para definir una variable multicriterio de tamaño, así como las dos variables que sirven para medir los resultados empresariales, esto es, la capacidad de financiación y la capacidad económica. La realización de estas técnicas se ha llevado a cabo mediante el programa estadístico SPSS para Windows.

\section{RESULTADOS}

La realización de un análisis de componentes principales nos ha servido para agrupar las variables objeto de estudio en un único componente para cada uno de los constructos: tamaño, capacidad de financiación, capacidad económica y capacidad mixta. Según se puede observar en la Tabla 3, el primer componente (componente 1) explica el $86 \%$ del total de la información contenida en las tres variables de tamaño iniciales y cuyo valor propio es superior a la unidad. La alta correlación existente entre las variables justifica el análisis y la elección del componente como variable representativa del tamaño (TAM).

Tabla 3. Análisis factorial de las variables de tamaño

\begin{tabular}{|c|c|c|c|c|c|c|c|c|c|}
\hline Tamaño & \multicolumn{3}{|c|}{ Matriz de Correlaciones } & \multicolumn{4}{c|}{ Varianza total explicada } & \multicolumn{2}{c|}{ Matriz componente 1 } \\
\hline & AT & FP & IE & C & Total & $\%$ Varianza & $\%$ acumulado & Variable & Valor \\
\hline AT & 1,000 & 0,577 & 0,611 & 1 & 2,167 & 72,232 & 72,232 & AT & 0,860 \\
\hline FP & 0,577 & 1,000 & 0,562 & 2 & 0,446 & 14,858 & 87,089 & FP & 0,836 \\
\hline IE & 0,611 & 0,562 & 1,000 & 3 & 0,387 & 12,911 & 100,00 & IE & 0,853 \\
\hline
\end{tabular}

Fuente: elaboración propia 
También se ha aplicado el análisis de componentes principales a cada uno de los constructos definidos para medir los resultados empresariales (capacidad financiera, capacidad económica y capacidad mixta). Sin embargo, en ninguno de estos casos se ha podido simplificar las variables elegidas para cada uno de dichos constructos, pues las correlaciones obtenidas han sido bajas y la explicación de la varianza del primer componente poco significativo. Así por ejemplo, en el caso del constructo capacidad financiera formada por cuatro variables (liquidez, solvencia, apalancamiento y estructura del apalancamiento), la explicación del primer componente es del 44,74\% y las correlaciones entre las variables son bajas. Del mismo modo, el constructo capacidad económica formado por tres variables (margen de ventas, rotación del activo y eficiencia) presenta unas correlaciones muy bajas y el componente 1 explica solo el 37,32\%. El constructo capacidad mixta muestra una correlación del $24 \%$ y el primer componente explica el $62 \%$ de la varianza.

Una vez realizada la agrupación de variables en componentes o factores y antes de realizar la estimación del modelo de regresión, vamos a analizar la dependencia mutua de las diferentes medidas de los resultados empresariales con la variable tamaño mediante el cálculo del coeficiente de correlación lineal de Spearman según muestra la Tabla 4. De ello se deduce que no existe una correlación positiva clara entre tamaño y resultados empresariales. Respecto a la capacidad financiera, dos de las variables muestran una relación negativa con el tamaño (solvencia y estructura del apalancamiento) y además, en una de ellas, la estructura del apalancamiento, presenta una relación significativa. En cuanto a la capacidad económica, dos de las tres variables utilizadas (margen de ventas y rotación del activo) muestran una relación negativa con el tamaño, si bien en este caso en ninguna de las variables se observa que la relación sea significativa (los valores del coeficiente están muy próximos al cero). La capacidad mixta presenta una relación positiva con el tamaño en las dos variables consideradas (ROA y ROE) y además en una de ellas, la rentabilidad de los recursos propios ROE, la relación es significativa.

Tabla 4. Contraste de Spearman

\begin{tabular}{|l|c|c|c|c|c|}
\hline \multicolumn{1}{|c|}{ Capacidad financiera } & TAM & L & S & AP & EA \\
\hline TAM & 1,000 & 0,141 & $-0,022$ & 0,055 & $-0,333^{*}$ \\
\hline Capacidad económica & TAM & MV & RA & E & \\
\hline TAM & 1,000 & $-0,014$ & $-0,029$ & 0,091 & \\
\hline Capacidad mixta & TAM & ROA & ROE & & \\
\hline TAM & 1,000 & 0,017 & $0,244^{*}$ & & \\
\hline
\end{tabular}

* La correlación es significativa al nivel 0,01 (bilateral).

Fuente: elaboración propia

Con el fin de cuantificar la relación entre el tamaño y los resultados empresariales, vamos a realizar una regresión mediante estimación por mínimos cuadrados ordinarios. La ecuación a estimar es: $\mathrm{RE}=\mathrm{B}_{0}+\mathrm{B}_{1}{ }^{*} \mathrm{TAM}$, donde $\mathrm{RE}$ es el resultado 
empresarial (variable dependiente) y TAM es el tamaño (variable independiente). Dado que los resultados empresariales se han medido a través de tres dimensiones diferentes (capacidad financiera, capacidad económica y capacidad mixta), se van a considerar tres ecuaciones diferentes:

1) $\mathrm{CF}=\mathrm{B}_{0}+\mathrm{B}_{1}{ }^{*} \mathrm{TAM}$, donde $\mathrm{CF}$ es la capacidad financiera medida a través de las variables liquidez, solvencia, apalancamiento y estructura del apalancamiento. Realizaremos cuatro regresiones lineales, una para cada variable.

2) $\mathrm{CE}=\mathrm{B}_{0}+\mathrm{B}_{1}$ * TAM, donde $\mathrm{CE}$ es la capacidad económica medida a través de las variables margen de ventas, rotación del activo y eficiencia. Realizaremos tres regresiones lineales, una para cada variable.

3) $\mathrm{CM}=\mathrm{B}_{0}+\mathrm{B}_{1}$ * TAM, donde CM es la capacidad mixta medida a través de las variables rentabilidad de los activos ROA y rentabilidad de los recursos propios ROE. Realizaremos dos regresiones lineales, una para cada variable.

En primer lugar, se realiza la regresión referente a la capacidad financiera con el tamaño con el fin de demostrar la Hipótesis 1. El tamaño influye sobre la capacidad de financiación de las cooperativas hortofrutícolas, de manera que las empresas de mayor tamaño presentan una mayor capacidad de financiación.

La Tabla 5 muestra los valores de los estadísticos, así como las medidas de $\mathrm{R}^{2} \mathrm{y}$ $\mathrm{F}$ de Snedecor para demostrar el grado de explicación del modelo para la regresión de la capacidad financiera. Según la $\mathrm{R}^{2}$, que indica la proporción de varianza explicada por el modelo al variar entre 0 y 1, ninguna de las variables consideradas permite una explicación del modelo, pues dichos valores están muy próximos al cero; solamente la variable estructura del apalancamiento muestra un valor algo mayor. Según la F de Snedecor, la única variable significativa es la estructura del apalancamiento, pues la significatividad de la F se sitúa por debajo de 0,05 (valor considerado como que el modelo es significativo). Según esta variable, la relación entre tamaño y capacidad financiera es negativa, dado que el coeficiente de la variable independiente toma valor negativo. Por tanto, rechazamos la hipótesis 1 según la cual el tamaño influye positivamente en la capacidad financiera de las cooperativas agrarias.

Tabla 5. Análisis de regresión para la capacidad financiera

\begin{tabular}{|l|c|c|c|c|c|c|c|c|}
\hline \multirow{2}{*}{} & \multicolumn{2}{|c|}{ Liquidez } & \multicolumn{2}{c|}{ Solvencia } & \multicolumn{2}{c|}{ Apalancamiento } & \multicolumn{2}{c|}{$\begin{array}{c}\text { Estructura del } \\
\text { Apalancamiento }\end{array}$} \\
\cline { 2 - 9 } & Beta & Sig. & Beta & Sig. & Beta & Sig. & Beta & Sig. \\
\hline Constante & 2,584 & 0,000 & 6,766 & 0,030 & 0,495 & 0,000 & 0,678 & 0,000 \\
\hline TAM & $-0,089$ & 0,786 & 0,189 & 0,951 & 0,025 & 0,423 & $-0,058$ & 0,026 \\
\hline $\mathrm{R}^{2}$ & \multicolumn{2}{|c|}{0,001} & \multicolumn{2}{c|}{0,000} & \multicolumn{2}{c|}{0,009} & 0,068 \\
\hline $\mathrm{R}^{2}$ corregida & $-0,013$ & \multicolumn{2}{c|}{$-0,014$} & \multicolumn{2}{c|}{$-0,005$} & 0,055 \\
\hline F de Snedecor & 0,074 & \multicolumn{2}{c|}{0,004} & \multicolumn{2}{c|}{0,648} & \multicolumn{2}{c|}{5,173} \\
\hline Signific. F & 0,786 & \multicolumn{2}{c|}{0,951} & \multicolumn{2}{c|}{0,423} & 0,026 \\
\hline
\end{tabular}

Fuente: elaboración propia 
En segundo lugar, se realiza la regresión referente a la capacidad económica con el tamaño con el fin de demostrar la Hipótesis 2. El tamaño influye sobre la capacidad de generar resultados de las cooperativas hortofrutícolas, de manera que las empresas de mayor tamaño presentan una mayor capacidad de generar resultados.

La Tabla 6 muestra los valores de los estadísticos, así como las medidas de $\mathrm{R}^{2} \mathrm{y}$ F de Snedecor para demostrar el grado de explicación del modelo para la regresión de la capacidad económica. Según la $\mathrm{R}^{2}$, que indica la proporción de varianza explicada por el modelo al variar entre 0 y 1 , ninguna de las variables consideradas permite una explicación del modelo, pues dichos valores están muy próximos al cero. Los resultados de la F de Snedecor coinciden, es decir, ninguna de las variables muestra una significatividad de la $\mathrm{F}$ suficiente pues todos los valores están muy por encima de 0,05. Por tanto, rechazamos la hipótesis 2 según la cual el tamaño influye positivamente en la capacidad económica de las cooperativas agrarias.

Tabla 6. Análisis de regresión para la capacidad económica

\begin{tabular}{|l|c|c|c|c|c|c|}
\hline \multirow{2}{*}{ Constante } & \multicolumn{2}{|c|}{ Margen de ventas } & \multicolumn{2}{c|}{ Rotación de activos } & \multicolumn{2}{c|}{ Eficiencia } \\
\cline { 2 - 7 } & Beta & Sig. & Beta & Sig. & Beta & Sig. \\
\hline TAM & 0,003 & 0,399 & 0,828 & 0,000 & $-8,105$ & 0,560 \\
\hline R $^{2}$ & $-0,001$ & 0,793 & $-0,039$ & 0,431 & 5,101 & 0,716 \\
\hline R $^{2}$ corregida & \multicolumn{2}{|c|}{0,001} & \multicolumn{2}{c|}{0,009} & \multicolumn{2}{c|}{0,002} \\
\hline F de Snedecor & \multicolumn{2}{|c|}{$-0,013$} & \multicolumn{2}{c|}{$-0,005$} & \multicolumn{2}{c|}{$-0,012$} \\
\hline Signific. F & \multicolumn{2}{|c|}{0,070} & \multicolumn{2}{c|}{0,626} & \multicolumn{2}{c|}{0,134} \\
\hline
\end{tabular}

Fuente: elaboración propia

En tercer lugar, se realiza la regresión referente a la capacidad mixta con el tamaño con el fin de demostrar la Hipótesis 3. El tamaño influye sobre la capacidad de influencia de la estructura económica y financiera para generar beneficios de las cooperativas hortofrutícolas, de manera que las empresas de mayor tamaño presentan una mayor capacidad de influencia.

La Tabla 7 muestra los valores de los estadísticos, así como las medidas de $\mathrm{R}^{2}$ y $\mathrm{F}$ de Snedecor para demostrar el grado de explicación del modelo para la regresión referente a la capacidad mixta. Según la $\mathrm{R}^{2}$, que indica la proporción de varianza explicada por el modelo al variar entre 0 y 1 , ninguna de las variables consideradas permite una explicación del modelo, pues dichos valores están muy próximos al cero; solamente la variable ROE muestra un valor algo mayor. Según la $\mathrm{F}$ de Snedecor, la única variable significativa es la ROE, pues la significatividad de la $\mathrm{F}$ se sitúa por debajo de 0,05 (valor considerado como que el modelo es significativo). Según esta variable, la relación entre tamaño y capacidad mixta es positiva, dado que el coeficiente de la variable independiente toma valor positivo. Por tanto, podemos aceptar en parte la hipótesis 3 según la cual el tamaño influye positivamente en la capacidad mixta de las cooperativas agrarias. 
Tabla 7. Análisis de regresión para la capacidad mixta

\begin{tabular}{|c|c|c|c|c|}
\hline \multirow{2}{*}{} & \multicolumn{2}{|c|}{ ROA } & \multicolumn{2}{c|}{ ROE } \\
\cline { 2 - 5 } & Beta & Sig. & Beta & Sig. \\
\hline Constante & 0,001 & 0,876 & 0,031 & 0,000 \\
\hline TAM & 0,001 & 0,619 & 0,016 & 0,034 \\
\hline $\mathrm{R}^{2}$ & \multicolumn{2}{|c|}{0,003} & \multicolumn{2}{|c|}{0,062} \\
\hline $\mathrm{R}^{2}$ corregida & \multicolumn{2}{|c|}{$-0,011$} & \multicolumn{2}{c|}{0,049} \\
\hline F de Snedecor & \multicolumn{2}{|c|}{0,249} & \multicolumn{2}{c|}{4,693} \\
\hline Signific. F & \multicolumn{2}{|c|}{0,619} & \multicolumn{2}{c|}{0,034} \\
\hline
\end{tabular}

Fuente: elaboración propia

Resumiendo, podemos explicar los resultados empresariales determinados por la capacidad financiera y la capacidad mixta a través de las siguientes ecuaciones. La dimensión capacidad económica no se puede explicar:

1) Capacidad financiera (estructura apalancamiento) $=0,678-0,058$ * Tamaño

2) Capacidad mixta (rentabilidad recursos propios) $=0,031+0,016$ * Tamaño

En definitiva, hemos de rechazar la hipótesis general según la cual el tamaño influye sobre los resultados empresariales de las cooperativas hortofrutícolas, de manera que las empresas de mayor tamaño presentan un mayor éxito organizativo.

\section{DISCUSIÓN Y CONCLUSIONES}

El éxito de una organización depende, en parte, de diferentes factores de contingencia o parámetros estructurales como el tamaño o la antigüedad, entre otros. Estos factores pueden contribuir al éxito empresarial, incluso en organizaciones donde en gran medida se sacrifica una parte de la rentabilidad económica por la rentabilidad social o rentabilidad de sus socios. Las cooperativas hortofrutícolas desarrollan una labor social importante dirigida a colectivos (pequeños agricultores) con un escaso poder de negociación frente a sus distribuidores, por lo que es necesario que estas organizaciones sobrevivan mediante la realización de una determinada actividad económica rentable. Aunque su papel en Europa es relevante, muchas de estas empresas se encuentran en una situación de debilidad que las puede llevar a la desaparición.

Desde algunos sectores profesionales y sobre todo académicos, se propone como solución a los problemas de las cooperativas agrarias, el aumento de su tamaño organizativo mediante operaciones de fusiones y adquisiciones. Sin embargo, la mayor parte de los estudios realizados no demuestran que exista una relación clara positiva entre rentabilidad y tamaño. 
El estudio empírico realizado tampoco es concluyente en la demostración de que el aumento de tamaño de las cooperativas agrarias suponga una mejora de los resultados económicos y financieros de estas entidades. Los resultados obtenidos demuestran que la capacidad económica de las cooperativas agrarias no depende en ningún caso del tamaño y que su capacidad financiera depende negativamente, es decir, que a mayor tamaño menor capacidad de financiación. Solamente en el caso de la rentabilidad de los recursos propios se ha demostrado que existe una relación positiva con el tamaño, si bien la significatividad del modelo tampoco es muy alta. Por tanto, el análisis empírico realizado demuestra que el aumento de tamaño no es una medida apropiada para resolver el problema de las cooperativas hortofrutícolas, por lo que habrá que buscar otro tipo de opciones estratégicas para resolver el principal problema que padecen, que es el de la falta de poder de negociación frente a las grandes empresas de la distribución comercial, que imponen los precios.

Es de interés la realización de nuevos estudios empíricos que analicen, no solamente como único factor de contingencia el tamaño, si no que introduzcan otro tipo de factores tanto internos (antigüedad, capacidad de gestión, etc.) como externos (grado de concentración del sector, poder de negociación de los clientes y de los proveedores, etc.), que sí podrían ser determinantes en el éxito de este tipo de empresas agrarias. Habría que realizar un estudio más amplio, que contemplara un número mayor de parámetros estructurales. Del mismo modo, también se deberían contemplar otras variables distintas para medir las diferentes dimensiones o constructos estudiados, sobre todo en lo que se refiere a los resultados empresariales, pero también al tamaño. También es interesante realizar un análisis comparativo con otras formas empresariales para comprobar si los niveles de influencia demostrados se cumplen también en el caso de otro tipo de organizaciones. 


\section{REFERENCIAS}

1. ADENAEUER, L.; HECKELEI, T. (2011). Foreign Direct investment and the performance of European Agrobusiness Firms. Journal of Agricultural Economics. Vol. 62, No. 3, pp. 639-654.

2. ARCAS, Narciso (2002). “Contribución de las cooperativas agrarias de segundo grado a la orientación al mercado de sus cooperativas asociadas: efectos en el desempeño de la relación". CIRIEC-España. Revista de Economía Pública, Social y Cooperativa. No. 41, pp. 139-162.

3. ARCAS, Narciso; GARCÍA PÉREZ DE LEMA, D.; GUZMÁN, I. (2006). “La eficiencia técnica como medida de rendimiento de las cooperativas agrarias". CIRIEC-España. Revista de Economía Pública, Social y Cooperativa. No. 55, pp. 289-311.

4. BAHAMONDE, E. (2000). Las Cooperativas Agrarias ante el Siglo XXI. Anuario de Estudios Cooperativos. No. 1, pp. 41-50.

5. BAREA, José; MONZÓN, José Luis (directores) (2002). La Economía social en España en el año 2000. CIRIEC-España. Valencia. 450p.

6. CERVERA, A. y BOHÓRQUEZ, M. (2000). "El asociacionismo como fuente de ventaja en el sector agroalimentario andaluz". REVESCO: Revista de Estudios Cooperativos. No. 70, pp. 31-46.

7. CLAVER-CORTÉS, Enrique; PERTUSA-OORTEGA, E. M.; MOLINA-AZORÍN, José Francisco (2011). “Estructura organizativa y resultado empresarial: un análisis empírico del papel mediador de la estrategia". Cuadernos de Economía y Dirección de la Empresa. No. 14, pp. 1-12.

8. COGECA (2010). Agricultural Cooperatives in Europe. European Agri-cooperatives COGECA, Brussels.

9. DEMESETZ, H. (1973). "Industry structure, market rivalry and public policy". Journal of Law and Economics. No. 16, pp. 1-10.

10. DOMINGO SANZ, Joaquín (2001)."Ratios para el análisis de rentabilidad de las cooperativas agrarias andaluzas". CIRIEC-España. Revista de Economía Pública, Social y Cooperativa. No.38, pp. 171-186.

11. FERNÁNDEZ ZAMUDIO, M. A.; CABALLERO, P.; DE MIGUEL, M. D. (2006).“'La gestión del minifundio a través de las cooperativas en la Comunidad Valenciana". CIRIEC-España. Revista de Economía Pública, Social y Cooperativa. No. 55, pp. 193-219.

12. FERRUZ, L.; GONZÁLEZ, J. (1994). “Tamaño y rentabilidad de la banca extranjera en España: 19861990". Actualidad Financiera. No. 46, pp. 655-672.

13. FILIPPI, M.;TRIBOULET, P. (2006).“Typologies des comportaments à innover des coopératives agricoles. Une étude en región Midi-Pyrénées". Economie Rurale. No. 296, pp. 20-38.

14. GALBRAITH, J.R. (2002). Desining organizations: an executive guide to strategy, structure and process. Jossey-Bass Business and Management series. San Francisco.

15. GALDEANO, E. (2006). "Productivity and efficiency analysis of horticultural co-operatives”. Spanish Journal of Agricultural Research. Vol. 4, No. 3, pp. 191-201. 
16. GEARY, J.K. (1999). "The new workplace: change at work in Ireland". The International Journal of Human Resource Management. Vol. 10, No. 5, pp. 870-890.

17. GIAGNOCAVO, Chyntia.; GEREZ, Sonia;VARGAS, Carlos; CAMPOS, Vanessa (2012). Support for Farmer's Cooperatives. Structure and strategy of fruit and vegetables cooperatives in Almeria and Valencia, Spain. Document of European Union. 110p.

18. GIBRAT, R. (1931). Les inégalités economiques. Dunod. Paris.

19. GONZÁLEZ PÉREZ, A. L.; CORREA, A. (1998). “Crecimiento y tamaño: un estudio empírico”. Revista Española de Financiación y Contabilidad. Vol. 27, No. 95, pp. 541-573.

20. GONZÁLEZ, J. I.; HERNÁNDEZ, M. C.; RODRÍGUEZ, T. (2000). “Contribución del tamaño y el sector en la explicación de la rentabilidad empresarial". Revista Española de Financiación y Contabilidad. Vol.29, No. 106, pp. 903-930.

21. JACQUEMIN, A.; GHELLINK, E. (1980). “Familial control, size and perfomance in the largest french firms". European Economic Review. No. 13, pp. 81-91.

22. JULIÁ, Juan Francisco; MARÍ, Sergio (2002): "Agricultura y desarrollo rural", CIRIEC-España. Revista de Economía Pública, Social y Cooperativa. No. 48, pp. 25-52.

23. KUMAR, M. S. (1985). Growth, Acquisitions and investment. Cambridge University Press. Cambridge.

24. MARÍ, Sergio; JULIÁ, Juan Francisco (2001).“Evolución del cooperativismo agrario en España. De los sindicatos agrícolas a la actualidad". REVESCO: Revista de Estudios Cooperativos. No. 73, pp. 59-80.

25. MELIÁ, Elena; JULIÁ, Juan Francisco (2008). “La intercooperación: una respuesta a las actuales demandas del cooperativismo agrario". Estudios de Economía Aplicada. Vol. 26, No. 1, pp. 57-88.

26. MELIÁ, Elena; JULIÁ, Juan Francisco; GARCÍA MARTÍNEZ, Gabriel (2007). “Análisis de la eficiencia de los procesos de fusión cooperativa en la Comunidad Valenciana". XIV Congreso AECA. Valencia, pp. 125-133.

27. MILES, R.E.; SNOW, C.C. (1978). Organizational Strategy, Structure and Process. McGraw-Hill. Tokio. $421 \mathrm{p}$.

28. MILES, R. E.; SNOMW, C.C. (1994). Fit, Failure and the hall of Fame. McMillan. New York. 259p.

29. MILLER, D. (1992). “Environmental fit versus internal fit”. Organization Science. Vol. 3, No. 2, pp. 159178.

30. MINTZBERG, Henry (1979). The structuring of organizations. Prentice-Hall. London. 550p.

31. MONTEGUT, Yolanda (2006). Análisis de los factores explicativos del éxito competitivo en las almazaras cooperativas catalanas. Universitat de Lleida. Lleida. 231p.

32. MONTEGUT, Yolanda; CRISTOBAL, Eduard (2010). “Análisis empresarial de las cooperativas del sector de la fruta dulce de Cataluña". REVESCO: Revista de Estudios Cooperativos. No. 101, pp. 28-57.

33. MONZÓN, José Luis (director) (2009). La Economía Social en España en el año 2008. CIRIEC- España. Valencia. 350p.

34. NADLER, D.; TUSHMAN, M. (1997). "A congruence model for organization problem solving". En Tushman, M. and Anderson, P. (eds.): Managing strategic innovation and change: organization, architectures and managing innovation. Oxford University Press. New York, pp. 159-171. 
35. PERTUSA-ORTEGA, E. M.; MOLINA-AZORÍN, J. F.; CLAVER-CORTÉS, Enrique (2008). "A comparative analysis of the influence that different fit perspectives have on firm performance". Management Research. No. 6, pp. 139-150.

36. PRAIS, S.J. (1976). The evolution of giant firms in Britain. Cambridge University Press. Cambridge. 222p.

37. ROJAS, J. L. (2007). "La internacionalización de las cooperativas agrarias castellano-manchegas". Boletín económico de ICE, Información Comercial Española. No. 2923, pp. 57-68.

38. RUIZ, M. C.; HERNÁNDEZ, M. J.; GARCÍA MARTÍ, E. (2006). “Estado actual de la investigación sobre sociedades cooperativas agrarias en España". CIRIEC-España. Revista de Economía Pública, Social y Cooperativa. No. 56, pp. 65-86.

39. RUMELT, R.P. (1991). "How much does industry matter?" Strategic Management Journal. No. 12, pp. 167-185.

40. SABATÉ, Pere (2002). "Análisis comparativo de la eficiencia de las cooperativas fruteras de la provincia de Lleida". CIRIEC-España. Revista de Economía Pública, Social y Cooperativa. No. 41, pp. 163-182.

41. SARASA, C. P.; RIVERA, L. M.; CAPÓ, J. (2009). "Análisis multicriterio de resultados y solvencia para cooperativas agroalimentarias valencianas (2003-2007)". En Meliá, E. and Server, R. (directs.): La gestión de empresas de economía social. Ed. Marcial Pons. Madrid, pp. 151-168.

42. SOBOH, R.; LANSINK, A. O.; VAN DIJK, G. (2012). "Efficiency of Cooperatives and investors owned firms revisited", Journal of Agricultural Economics, 63 (1), 142-157.

43. VALENTINOV, V. (2007)."Why are cooperatives important in agriculture? An Organizacional Economics Perspective". Journal of Institutional Economics. No. 3, pp. 55-69.

44. VIDAL, F. (1999). Eficiencia económica de las cooperativas de comercialización hortofrutícola de la comunidad valenciana. Universidad Politécnica de Valencia. Valencia. 231p.

45. YOUNDT, M. A. et al. (1996). Human Resource Management Manufacturing Strategy and Firm Performance. Academy of Management Journal. Vol. 39, No. 4, pp. 836-866. 